\title{
A homogeneous time-resolved fluorescence-based high-throughput screening for discovery of inhibitors of Nef-sdAb19 interaction
}

\author{
XIAOQIN FAN $^{1,3^{*}}$, JINMEI WEI $^{1 *}$, HAITING XIONG $^{1}$, XIAOHUI LIU ${ }^{1}$, \\ SERGE BENICHOU ${ }^{2}$, XUEJUAN GAO ${ }^{1}$ and LANGXIA LIU ${ }^{1}$
}

\author{
${ }^{1}$ Key Laboratory of Functional Protein Research of Guangdong Higher Education Institutes, Institute of Life and \\ Health Engineering, Jinan University, Guangzhou 510632, P.R. China; ${ }^{2}$ Cochin Institute, INSERM U1016, \\ Centre National de la Recherche Scientifique UMR8104, Université Paris-Descartes, Paris, France
}

Received June 11, 2015; Accepted July 28, 2015

DOI: $10.3892 /$ ijo.2015.3132

\begin{abstract}
The human immunodeficiency virus (HIV) protein negative factor (Nef) is important for AIDS pathogenesis. An anti-Nef single-domain antibody (sdAb19) derived from camelids has been previously generated and shown to effectively block the physiological functions of Nef in vitro and in vivo in $n e$-transgenic mice. However, sdAb19 must be ectopically expressed within the target cell to be able to exert its neutralizing effect on Nef, while the extra-cellular administration method turned out to be ineffective. This might suggest a default of the stability or/and deliverability of sdAb19. The identification of small molecule compounds capable of inhibiting the Nef-sdAb19 interaction and mimicking the neutralizing activity of sdAb19 in vivo would therefore be the means of circumventing the problem encountered with sdAb19. Here we describe the development of a high-throughput screening method combining the homogeneous time-resolved fluorescence (HTRF) and the microscale thermophoresis (MST)
\end{abstract}

Correspondence to: Dr Xuejuan Gao or Professor Langxia Liu, Key Laboratory of Functional Protein Research of Guangdong Higher Education Institutes, Institute of Life and Health Engineering, Jinan University, Guangzhou 510632, P.R. China

E-mail: tgaoxj@jnu.edu.cn

E-mail: langxialiu@gmail.com

Present address: ${ }^{3}$ Longgang Central Hospital, ENT Hospital, Shenzhen ENT Institute, Shenzhen 518116, P.R. China

*Contributed equally

Abbreviations: HTRF, homogeneous time-resolved fluorescence; MST, microscale thermophoresis; sdAb, single-domain antibodies; HIV, human immunodeficiency virus; AIDS, acquired immune deficiency syndrome; FRET, fluorescence resonance energy transfer technology

Key words: homogeneous time-resolved fluorescence, microscale thermophoresis, Nef, single doman antibody, small molecule compound techniques for the identification of small-molecule compounds inhibiting the Nef-sdAb19 interaction by binding to Nef protein. Eight small-molecule compounds have been selected for their ability to significantly inhibit the Nef-sdAb19 interaction and to bind to Nef. These molecules could be further assessed for their potential of being the Nef-neutralizing agents in the future.

\section{Introduction}

Despite the considerable progress made in the field of anti-HIV drug development, the acquired immune deficiency syndrome (AIDS) is still a serious health concern across the world since its discovery more than thirty years ago. Due to its high mutation rate, HIV is often able to evade the host immune system, and also the antiviral therapies $(1,2)$. Therefore, the development of novel drugs and therapeutic methods against the virus is important. The HIV accessory proteins represent the alternative targets for anti-HIV drug development. In particular, the negative factor (Nef), an accessory protein of HIV which plays a critical role in the physio-pathogenesis of AIDS could be a good target for the drug research and development. Nef is expressed early in the viral life cycle and targeted to the plasma membrane $(3,4)$. It contains an $\mathrm{N}$-myristoylation cytoplasmic region that is required for its association with cellular membranes and virtually critical for its complex biological activities. Nef exists as homodimers in cells, and all types of Nef are conserved with six structured core domains which forms one $\alpha$-helix and five $\beta$-layer structures (5). The flexible regions of Nef, such as its large surfaces accessible for interactions and important conformational changes, are involved in increasing infectious viral particles, activating CD4 lymphocytes and preventing infected cells from apoptosis (6-8).

Recently, antibody therapy has obtained remarkable results. The single-domain antibodies (sdAb) derived from camelids is a major breakthrough in the development of antibody therapy $(5,9,10)$. The sdAb19, consisting only of a single variable domain which recognizes the antigenic epitope of the target protein, is relatively small with a molecular weight of $13 \mathrm{kDa}$, and can penetrate in cavities located on the surface of antigens. Previous studies have demonstrated 
the sdAb fragments displayed efficient therapeutic activity in many diseases such as cancer, neurodegenerative diseases and rheumatoid arthritis (11-13). The sdAb19 generated by Bouchet et al targeted the HIV Nef protein with a high affinity and inhibited biologic activities of Nef both in vitro and in vivo in a nef-transgenic mouse model, suggesting that inhibition of Nef function is a potential method to block HIV (9). However, sdAb19 must be ectopically expressed via an expression vector within the target cell to be able to exert its neutralizing effect on Nef, while the extra-cellular administration method turned out to be ineffective. This might suggest a default of the stability or/and deliverability of sdAb19. The identification of small molecule compounds capable of mimicking the neutralizing activity of sdAb19 and displaying better stability or/and deliverability in vivo would therefore be the means of circumventing the problem encountered with sdAb19. In this study, we took advantage of the homogeneous time-resolved fluorescence (HTRF) technology to carry out a high-throughput screening of a library of small molecules. HTRF technology is an ideal platform used for drug discovery in high-throughput screening, which combines fluorescence resonance energy transfer technology (FRET) with timeresolved measurement (TR). The time-resolved characteristic of HTRF technology allows for the removal of nearly all environmental and compound interference effects. As a sensitive and reliable method due to its reduced inter-well variation and fluorescence interference, this technology has been developed for many antibody-based assays including GPCR signaling, kinases, cytokines and biomarkers, bioprocess, as well as the assay for biomolecular interaction (14-18). We selected the molecules capable of inhibiting the Nef-sdAb19 interaction by this method, then used the microscale thermophoresis (MST) technique to identify, among these candidate molecules, those that fix on Nef. These are the molecules which probably inhibit the Nef-sdAb19 interaction by competition of Nef binding with sdAb19, and could possibly mimic the anti-Nef activity in vivo.

\section{Materials and methods}

Antibodies and chemicals. Eu ${ }^{3+}$ cryptate-conjugated anti-GST antibody (anti-GST-Eu) and XL665-conjugated anti-6xHis antibody (anti-6xHis-XL665) were purchased from Perkin-Elmer (France). Anti-his monoclonal antibody was purchased from Santa Cruz Biotechnology, Inc. (USA). Tris, sodium dodecyl sulfate (SDS) and glycine were obtained from Bio-Rad (USA). Protein inhibitor was purchased from Merk (USA). Isopropyl-1-thio- $\beta$-D-galactopyranoside (IPTG), lysozyme, imidazole and reduced glutathione were purchased from Applygen (China). Glutathione-Sepharose 4B beads were purchased from GE (USA), whereas $\mathrm{Ni}^{+}$beads from Qiagen N.V. (China). Pharmacologically active compounds (LOPAC) were from Sigma (USA). Pierce BCA Protein Assay kit was purchased from Thermo (USA).

Expression and purification of recombinant proteins. The pGEX-4T-Nef construct encoding GST-Nef and pcDNAsdAb19 expressing His-sdAb19 were transformed into Escherichia coli BL21. The expression of recombinant protein was induced by the addition of $0.2 \mathrm{mM}$ IPTG for 6-8 $\mathrm{h}$ at room temperature (RT) when transformed bacteria were grown to an OD600 of 0.6-0.8. The cells were collected and lysed by sonication in EBC buffer $(50 \mathrm{mM}$ Tris- $\mathrm{HCl}, 150 \mathrm{mM} \mathrm{NaCl}$, $1 \%$ Triton X-100, pH 7.6) containing $1 \mathrm{mM}$ phenylmethylsufonyl fluoride (PMSF), 1X complete mini EDTA-free protein inhibitors and $10 \mu \mathrm{g} / \mathrm{ml}$ lysozyme. Bacterial extracts were sonicated for $20 \mathrm{~min}$ and centrifuged at $12,000 \mathrm{rpm}$ for $30 \mathrm{~min}$ at $4^{\circ} \mathrm{C}$ to remove cell debris. GST-proteins were purified using Glutathione Sepharose 4B (GE Healthcare), and His-sdAb19 proteins were purified from supernatant using Ni-NTA Superflow (Qiagen). Glutathione and imidazole were excluded from the eluted proteins using $10 \mathrm{kDa}$-ultrafiltration (Millipore, USA). The concentration of protein was measured by BCA assay (Beyotime Biotechnology, China). The purity of recombinant proteins were determined by SDS-PAGE and coomassie blue straining (CBB).

Digestion by thrombin. Thrombin was added to digest the GST-Nef beads for $16 \mathrm{~h}$ at $4^{\circ} \mathrm{C}$. The digestion mix was then centrifuged at $12,000 \mathrm{rpm}$ for $5 \mathrm{~min}$ at $4^{\circ} \mathrm{C}$ to collect the supernatant.

GST pull-down assay. Equal amounts of GST-beads and GST-Nef-beads were incubated in EBC buffer with $500 \mu \mathrm{g}$ purified recombinant His-sdAb19 for $6 \mathrm{~h}$ at $4^{\circ} \mathrm{C}$. The beads were recovered by centrifugation at $1,000 \mathrm{rpm}$ for $2 \mathrm{~min}$ at $4^{\circ} \mathrm{C}$ and washed three times with $\mathrm{EBC}$ buffer, and proteins were eluted into $20 \mu 1$ of SDS sample buffer by heating to $95^{\circ} \mathrm{C}$ for 5 min. Proteins were separated using 10\% SDS-PAGE gels and then eletro-blotted to PVDF membrane. Membranes were blocked with 5\% non-fat milk for $1 \mathrm{~h}$ at RT, and then incubated with anti-His mouse antibody overnight at $4^{\circ} \mathrm{C}$. After washing, the blots were probed with horseradish peroxidase-conjugated anti-mouse secondary antibody (ProteinTech Group) for $1 \mathrm{~h}$ at RT, and then signals were visualized using ECL detection system (Beyotime Biotechnology).

Optimization of an HTRF assay for Nef-sdAb19 interaction. The following experiments were designed to determine the optimal concentration of HTRF reagents, including GST-Nef, His-sdAb19, anti-GST-Eu antibody, and anti-6xHis-XL665 antibody. i) The incubation of GST-Nef having a concentration in a range from 0 to $100 \mathrm{nM}$ with $100 \mathrm{nM}$ His-sdAb19, $5 \mathrm{nM}$ anti-GST-Eu antibody, and $100 \mathrm{nM}$ anti-6xHis-XL665 antibody was to determine the optimal concentration of GST-Nef through HTRF assay. ii) The incubation of His-sdAb19 having a concentration in a range from 0 to $100 \mathrm{nM}$ with $100 \mathrm{nM}$ GST-Nef, $5 \mathrm{nM}$ antiGST-Eu, and $100 \mathrm{nM}$ anti-6xHis-XL665 antibody was to determine the optimal concentration of His-sdAb19 through HTRF assay. iii) Anti-GST-Eu antibody, with the changeable concentration from 0 to $5 \mathrm{nM}$, co-incubated with $100 \mathrm{nM}$ anti-6xHis-XL665 antibody, $100 \mathrm{nM}$ GST-Nef, and $100 \mathrm{nM}$ His-sdAb19 was to determine the optimal concentration of $\mathrm{Eu}^{3+}$-GST antibody. iv) Anti-6xHis-XL665 antibody, with the increasing concentrations from 0 to $100 \mathrm{nM}$, co-incubated with $5 \mathrm{nM}$ anti-GST-Eu antibody, $100 \mathrm{nM}$ GST-Nef, and $100 \mathrm{nM}$ His-sdAb19 was to determine the optimal concentration of XL665-His antibody. During these HTRF assays, GST-Nef was incubated for $4 \mathrm{~h}$ at $4^{\circ} \mathrm{C}$, and then anti- 
A

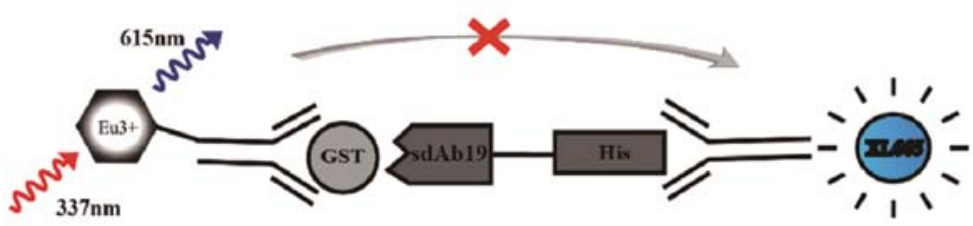

B

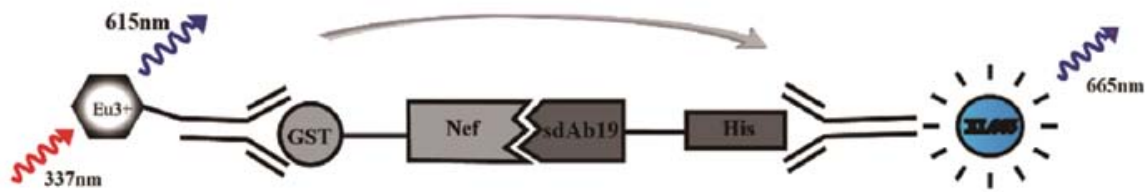

Figure 1. The schematic for homogeneous time resolved fluorescence assay. HTRF principle with Eu ${ }^{3+}$ and XL665 respectively as donor and acceptor. If the labeled GST-Nef binds to His-sdAb19 and each other, the two fluorephores will be in close proximity to allow FRET to occur, which result in a specific longlived fluorescence emission of XL665 at $665 \mathrm{~nm}$.

GST-Eu and anti-6xHis-XL665 antibodies were added to incubate away from light for $2 \mathrm{~h}$ at RT. The corresponding GST protein was used as negative control for all the tests. All $\mathrm{HTRF}$ reagents were diluted with Tris- $\mathrm{HCl}$ buffer and added to the 384-well plates to a final volume of $50 \mu 1$. Plates were stored in the dark at RT before reading using Perkin-Elmer VICTOR $^{\mathrm{TM}}$ X5 (Perkin-Elmer, USA). The excitation fluorescence of $337 \mathrm{~nm}$ was used to excite $\mathrm{Eu}^{3+}$ and the fluorescence emission at 665 and $615 \mathrm{~nm}$ were detected simultaneously. Signal was expressed in terms of HTRF ratio $\Delta F \%$, as the previous description (19). $\mathrm{R}=$ [signal 665/signal 615]*10,000; $\Delta \mathrm{R}=\mathrm{R}_{\mathrm{Nef}}-\mathrm{R}_{\mathrm{GST}} ; \Delta \mathrm{F} \%=(\Delta \mathrm{R} / \mathrm{RGST}) * 100$ (Fig. 1).

Nef-sdAb19 interaction inhibition assay. The library of pharmacologically active compounds (LOPAC, SigmaAldrich, USA) contains 1,280 well-characterized chemical compounds. The compounds of LOPAC library are stored at a concentration of $10 \mathrm{mM}$, dissolved in DMSO in 96-well plates at $-20^{\circ} \mathrm{C}$. Before the experiments, we diluted the compounds with the following procedure. Each compound $(5 \mu 1)$ was mixed with $95 \mu 1$ DMSO in other 96-well plates. Each diluted compound $(10 \mu \mathrm{l})$ was used for high-throughput screening and added into the 384-well black plate. Then, $10 \mu 1$ of $250 \mathrm{nM}$ GST-Nef (a final concentration of $50 \mathrm{nM}$ ) was incubated with the compounds for $2 \mathrm{~h}$ at $4^{\circ} \mathrm{C}$, following by incubated with $10 \mu \mathrm{l}$ of $100 \mathrm{nM}$ His-sdAb19 (a final concentration of $20 \mathrm{nM}$ ) for $4 \mathrm{~h}$ at $4^{\circ} \mathrm{C}$. Ten microliters of the donor fluorophore $\mathrm{Eu}^{3+}$ cryptate-conjugated anti-GST antibody (a final concentration of $2.5 \mathrm{nM}$ ) and $10 \mu \mathrm{l}$ of the acceptor fluorophore XL665conjugated anti-6xHis antibody (a final concentration of $100 \mathrm{nM}$ ) were added and incubated in dark condition for $2 \mathrm{~h}$ at RT. The GST protein was used as comparison substance. The total volume of the reaction was $50 \mu \mathrm{l}$. There were 3 multiple pores in every dosage group and negative control group. Plates were read by Perkin-Elmer VICTOR ${ }^{\mathrm{TM}}$ X5 by measurement of fluorescence emission at 665 and $615 \mathrm{~nm}$. The percentage of interaction inhibition was analyzed according to the following formula (19). Inhibition $(\%)=\left(\left(\mathrm{F}_{\mathrm{Nef}}-\mathrm{F}_{\mathrm{GST}}\right)-\left(\mathrm{F}_{\mathrm{com}}-\mathrm{F}_{\mathrm{GST}}\right)\right) /$ $\left(\mathrm{F}_{\mathrm{Nef}}-\mathrm{F}_{\mathrm{GST}}\right) * 100 ; \mathrm{F}=[$ signal $665 /$ signal 615$] * 10,000 . \mathrm{F}_{\mathrm{Nef}}$ is the fluorescence emission ration of GST-Nef group treated with DMSO; $\mathrm{F}_{\text {com }}$ is the fluorescence emission ration of GST-Nef group treated with the small molecular compound; $F_{\mathrm{GST}}$ is fluorescence emission ration of GST negative control group treated with DMSO; $\mathrm{F}$ is fluorescence emission ration of $665 \mathrm{~nm} / 615 \mathrm{~nm}$.

Excluding the false-positive reaction. Microscale thermophoresis (MST) was used to determine the binding affinities between Nef and the cadidates of HTRF. a fixed concentration of $200 \mathrm{nM}$ Nef, the candidates which were titrated with 122.07 to $25,000 \mathrm{nM}$ in PBS buffer. In order to allow binding, samples were incubated at least $30 \mathrm{~min}$ at room temperature followed by centrifugation for $5 \mathrm{~min}$ at $15,000 \mathrm{~g}$ to eliminate potential precipitates. Experimental measurements were performed in standard or hydrophilic capillaries using a NanoTemper Monolite $^{\text {TM }}$ NT.115 instrument (NanoTemper Technologies $\mathrm{GmbH}$, Germany) for red fluorescence dye. Thermophoresis signals for each of the 12 capillaries were monitored, which harbor different ratios of binding partners. The normalized fluorescence at a given time point was plotted against the concentration of unlabed candidates. The resulting sigmoidal curves were normalized, and each data point was determined. Data points were finally fitted by the Hill slope, and KD value were obtained. Kd Formula (law of mass action): $\mathrm{F}(\mathrm{c})=$ unbound + (bound-unbound $) / 2 *$ (FluoConc $+\mathrm{c}+\mathrm{Kd}-$ Sqrt ((FluoConc $+\mathrm{c}+\mathrm{Kd})^{\wedge} 2-4 *$ FluoConc*c) (20).

\section{Results}

Expression and purification of GST-Nef and His-sdAb19. The plasmids pGEX-4T-Nef and pcDNA-sdAb19 were transformed into E. coli BL21, and the recombinant bacteria was induced by IPTG in concentration of $0.2 \mathrm{mM}$ for $6-8 \mathrm{~h}$ at RT. As shown in Fig. 2, SDS-PAGE analysis showed that GST-Nef and His-sdAb19 were expressed in inclusion bodies in the E. coli cells and their molecular weights were $\sim 54$ and $27 \mathrm{kDa}$ (Fig. 2). The two recombinant proteins were purified with Glutathione-Sepharose 4B beads and Ni-NTA Superflow, 
A

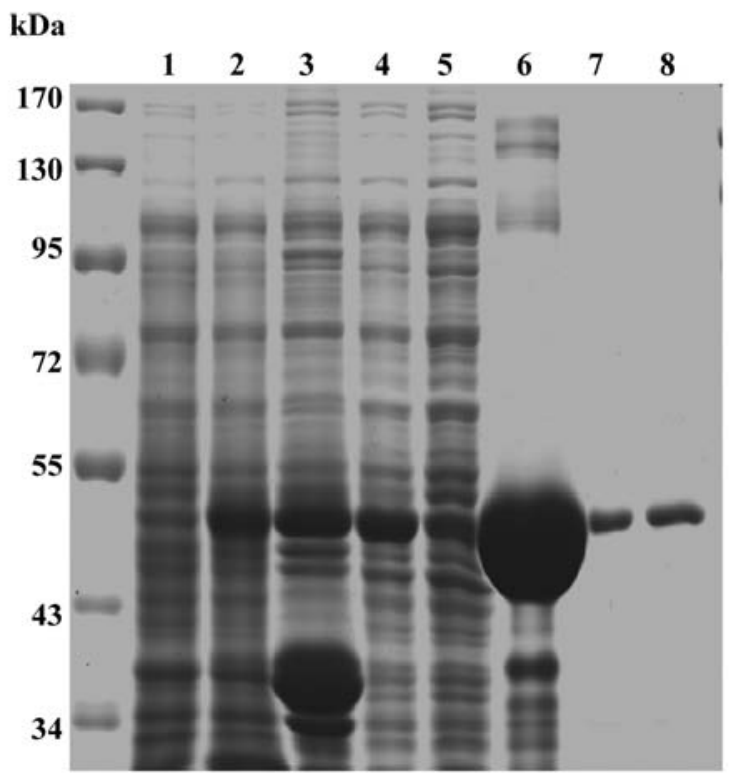

B

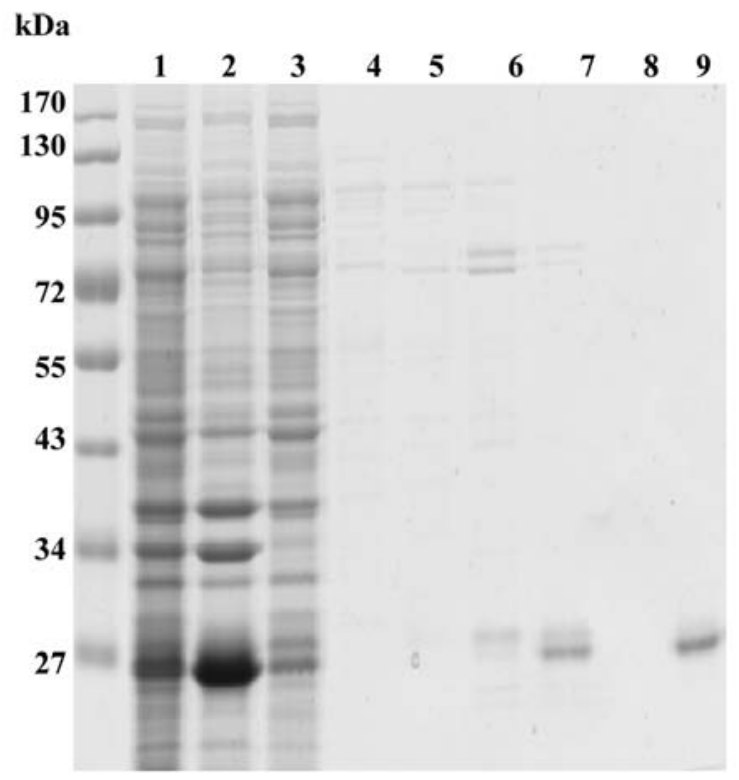

Figure 2. Expression and purification of recombinant GST-Nef and His-sdAb19. (A) The expression and purification of recombinant GST-Nef were analyzed by SDS-PAGE. Lane 1, E. coli BL21 transformed with pGEX-Nef without IPTG induction; lane 2, E. coli BL21 transformed with pGEX-Nef with IPTG induction; lane 3, the supernatant of cell lysates after IPTG induction; lane 4, the precipitation of cell lysates; lane 5, the supernatant of the flow-through; lane 6 , GST-Nef recombinant protein-beads before elution; lane 7, GST-Nef recombinant protein-beads after elution with $5 \mathrm{mM}$ reduced glutathione; lane 8, GST-Nef recombinant protein-beads after elution with $5 \mathrm{mM}$ reduced glutathione. (B) The expression and purification of recombinant His-sdAb19 were analyzed by SDA-PAGE. Lane 1, E. coli BL21 transformed with pET-sdAb19 without IPTG induction; lane 2, E. coli BL21 transformed with pET-sdAb19 with IPTG induction; lane 3, the supernatant of cell lysates after IPTG induction; lane 4, the precipitation of cell lysates; lane 5, the supernatant of the flow-through; lane 6 , His-sdAb19 recombinant protein-beads before elution; lane 7, His-sdAb19 recombinant protein-beads after elution with $10 \mathrm{mM}$ imidazole; lane 8, His-sdAb19 recombinant protein-beads after elution with $50 \mathrm{mM}$ imidazole; lane 9, His-sdAb19 recombinant protein-beads after elution with $100 \mathrm{mM}$ imidazole.

respectively. SDS-PAGE analysis showed that there was a single band at the expected position, indicating that the proteins had been purified successfully (Fig. 2).

In vitro interaction between GST-Nef and His-sdAb19. To demonstrate the direct interaction between Nef and sdAb19 in vitro, we performed an in vitro GST pull-down experiment using GST-Nef and His-sdAb19 recombinant proteins. Fig. 3 showed that His-sdAb19 could be pulled down by the immobilized GST-Nef but not the immobilized GST, indicating the direct and specific binding of GST-Nef and His-sdAb19 exists in an acellular context.

Optimization of HTRF assay reagents. To make sure that the interaction of Nef with sdAb19 (presented as HTRF ratio $\Delta \mathrm{F} \%$ ) responds sensitively to any interference coming from the small molecular inhibitors, the used concentration of four reagents must be optimized, including GST-Nef, His-sd Ab19, anti-GST-Eu antibody, and anti-6xHis-XL665 antibody. As described in detail in Materials and methods, with excessive His-sdAb19 (100 nM), anti-GST-Eu antibody $(5 \mathrm{nM})$, and anti-6xHis-XL665 antibody (100 nM), GST-Nef proteins having a concentration in a range from 0 to $100 \mathrm{nM}$ were added for reactions. GST proteins were used as negative control. The result showed that the observed interaction (HTRF ratio $\triangle \mathrm{F} \%$ ) increased linearly at the concentration of GST-Nef $<50 \mathrm{nM}$, while the observed interaction (HTRF ratio $\Delta \mathrm{F} \%$ ) decreased (Fig. $4 \mathrm{~A}$ ) at its concentration $>50 \mathrm{nM}$, suggesting the optimal concentration of GST-Nef is $\sim 50 \mathrm{nM}$.

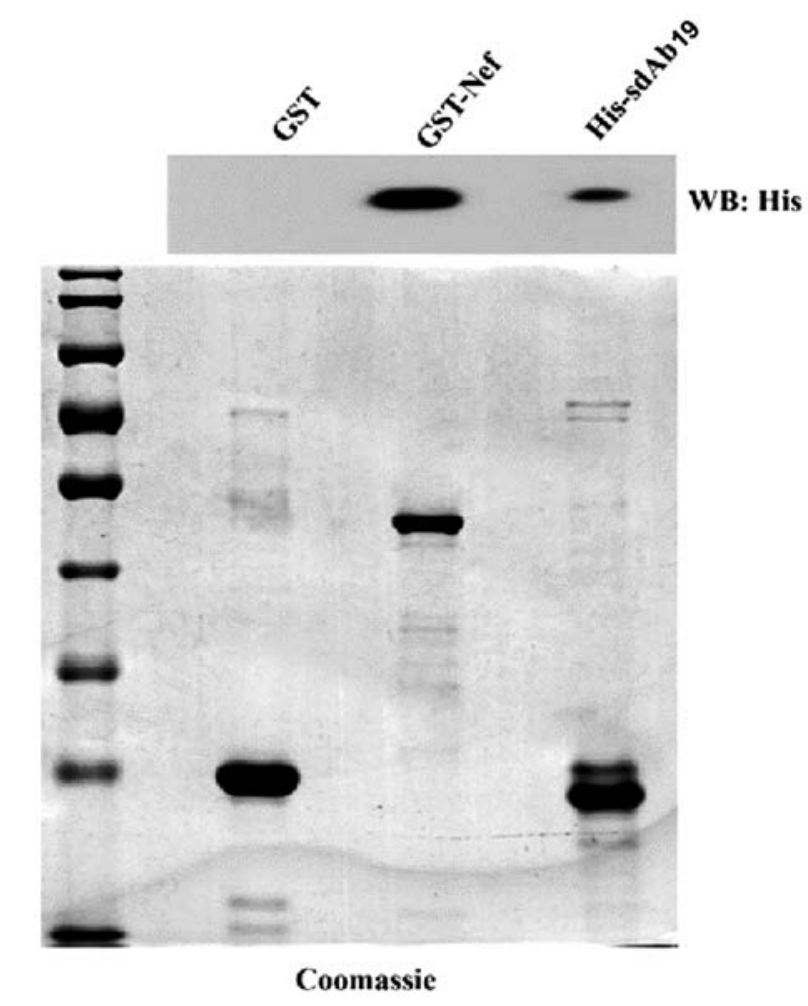

Figure 3. The direct interaction between Nef and sdAb19 determined by GST pull-down. Purified GST (lane 1) or GST-Nef recombinant protein (lane 2) were immobilized on Sepharose-Glutathione beads and incubated with purified His-sdAb19 protein. Lane 3 represents the input of His-sdAb19. The amounts of GST and GST-Nef used in the assays were checked by coomassie blue staining (lower panel) 
A

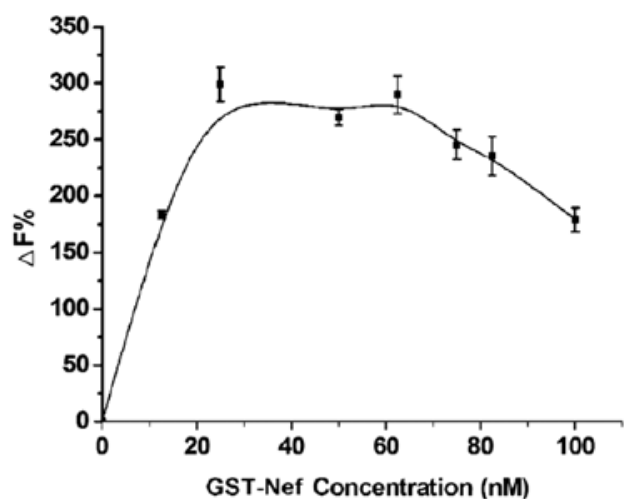

C

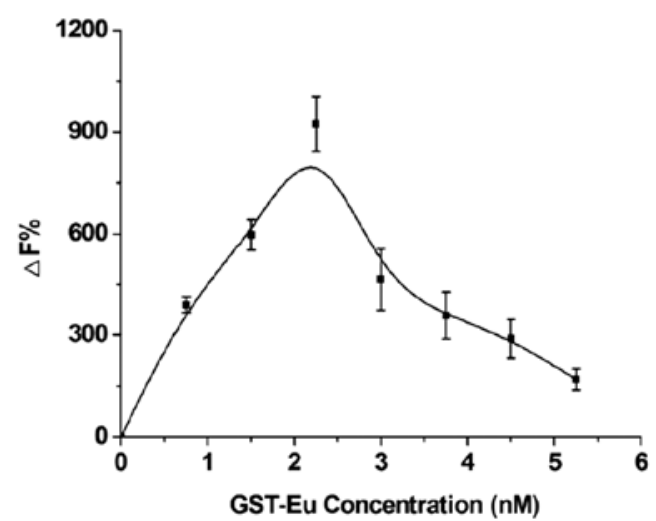

B
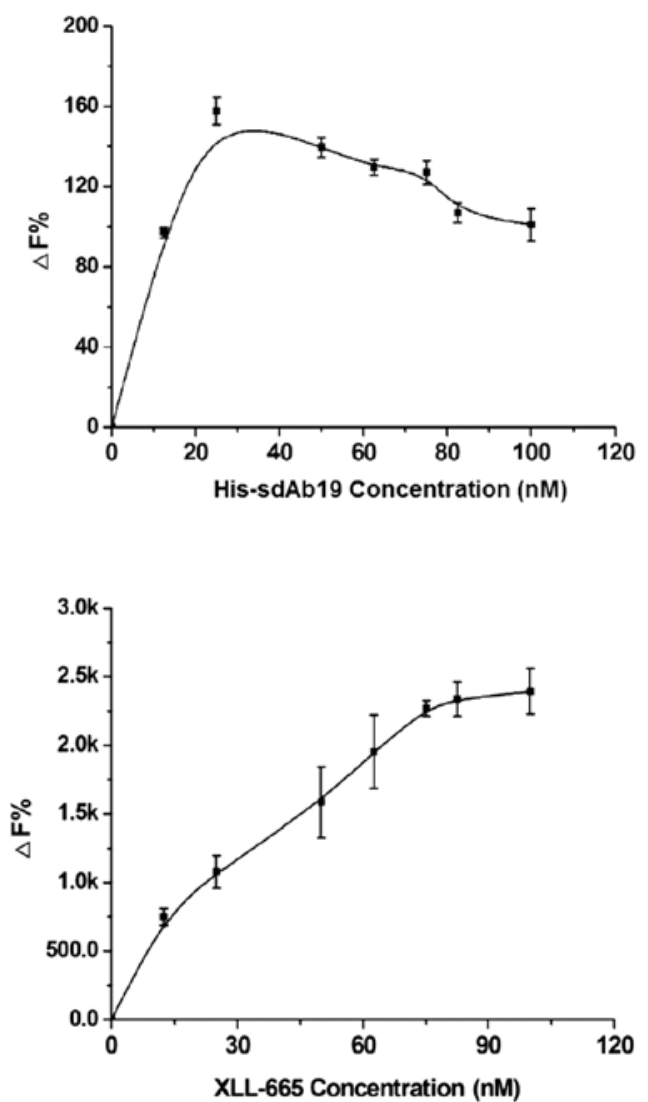

Figure 4. Optimization of HTRF assay reagents. (A) Optimization of the concentrations of GST-Nef for high-throughput screening. His-sdAb19 (100 nM), anti-GST-Eu antibody $(5 \mathrm{nM})$, and anti-6xHis-XL665 antibody $(100 \mathrm{nM})$ were mixed with different concentrations of GST-Nef from 0 to $100 \mathrm{nM}$ in black 384-wells. GST-Nef and His-sdAb19 were incubated for $4 \mathrm{~h}$ at $4^{\circ} \mathrm{C}$, then the anti-GST-Eu and anti-6xHis-XL665 antibodies were added for 2-h incubation at RT. Plates were read using Perkin-Elmer VICTOR X5. HTRF ratio $\Delta F \%$ is positively correlated with the Nef-sdAb19 interaction. (B) Optimization of the concentrations of His-sdAb19 for high-throughput screening. GST-Nef (100 nM), anti-GST-Eu antibody (5 nM), and anti-6xHis-XL665 antibody $(100 \mathrm{nM})$ were mixed with different concentrations of His-sdAb19 from 0 to $100 \mathrm{nM}$ in the black 384-wells, followed by a similar procedure of HTRF assay. (C) Determination of the concentrations of anti-GST-Eu antibody for high-throughput screening. GST-Nef (100 nM), His-sdAb19 (100 nM), and anti-6xHisXL665 antibody $(100 \mathrm{nM})$ were mixed with different concentrations of anti-GST-Eu antibody from 0 to $5 \mathrm{nM}$ in the black 384-wells, followed by a similar procedure of the HTRF assay. (D) Determination of the concentrations of anti-6xHis-XL665 antibody for high-throughput screening. GST-Nef (100 nM), His-sdAb19 $(100 \mathrm{nM})$, and anti-GST-Eu antibody $(5 \mathrm{nM})$ were mixed with different concentrations of anti-6xHis-XL665 antibody from 0 to $100 \mathrm{nM}$ in the black 384-wells, followed by a similar procedure of the HTRF assay.

Subsequently, the proper concentration of His-sdAb19 was determined by HTRF assay by the addition of enough GST-Nef (100 nM), anti-GST-Eu antibody (5 $\mathrm{nM})$, and anti-6xHis-XL665 antibody (100 nM) with His-sdAb19 (the concentrations from 0 to $100 \mathrm{nM}$ ). The result showed that the strongest interaction between GST-Nef and His-sdAb19 was observed when the concentrations of His-sdAb19 reached $40 \mathrm{nM}$ (Fig. 4B). Due to the fact that $\Delta \mathrm{F} \%$ of $20 \mathrm{nM}$ is almost half of $40 \mathrm{nM}$, and that $100 \mathrm{nM}$ GST-Nef was used in this reaction twice, than the chosen $50 \mathrm{nM}$ for screening, we decided on the concentration of $20 \mathrm{nM}$ for the following high-throughput screening. Whereas, GST at concentration of $100 \mathrm{nM}$ was the negative control.

Similarly, the suitable concentration of anti-GST-Eu antibody was detected with enough GST-Nef (100 nM), His-sdAb19 (100 nM), and anti-6xHis-XL665 antibody $(100 \mathrm{nM})$ mixed together with anti-GST-Eu antibody with the concentrations from 0 to $5 \mathrm{nM}$ for HTRF detection. The

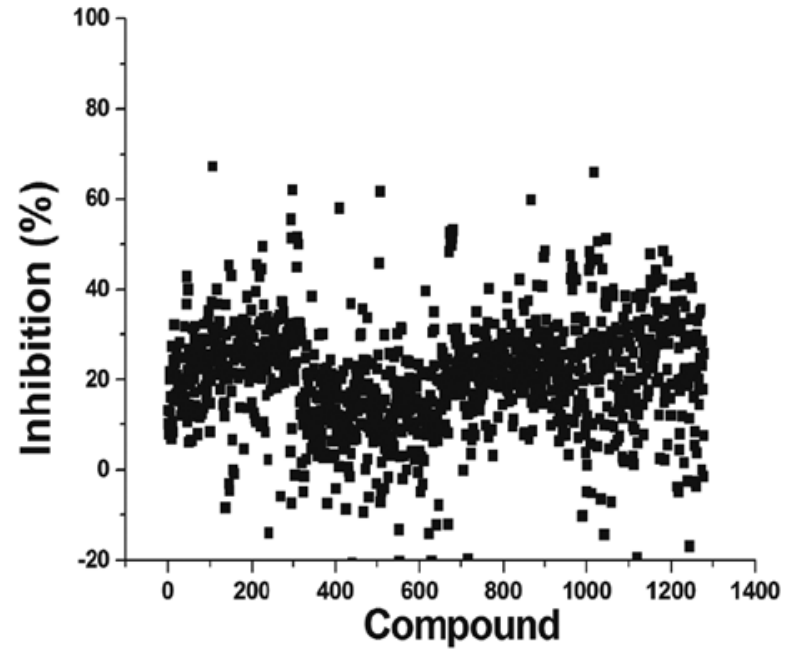

Figure 5. High-throughput screening of the small molecular inhibitors against Nef-sdAb19 interaction. Small box shows the inhibition (\%) of the screened compounds. 
Table I. The candidates compounds screened with HTRF and MST assay.

\begin{tabular}{rlllr}
\hline No. & \multicolumn{1}{c}{ Name } & \multicolumn{1}{c}{ Class } & HTRF & MST \\
\hline 1 & Trovafloxaxin mesylate & Immunomodulators and antibodies & + & + \\
2 & CL316243 & Adrenoceptor & + & + \\
3 & Felodipine & $\mathrm{Ca}^{2+}$ channel & + & + \\
4 & R(+)-IAA-94 & $\mathrm{Cl}^{-}$channel & + & + \\
5 & Loratadine & Histamine & + & + \\
6 & Isoliquiritigenin & Cyclic nucleotides & + & + \\
7 & IC261 & Phosphorylation & + & + \\
8 & A3 hydrochloride & Phosphorylation & + & + \\
9 & Cystamine & Glutamate & + & - \\
10 & Artemether & Immunomodulators and antibiotics & + & - \\
11 & Colchicine & Cytoskeleton and ECM & + & - \\
12 & CNS-1102 & Glutamatc & + & - \\
13 & Enoximone & Cyclic nucleotides & + & - \\
14 & Ketorolac tris salt & Prostaglandin & + & - \\
15 & Niclosamide & Antibiotic & + & - \\
16 & Auranofin & Phosphorylation & & - \\
\hline
\end{tabular}

HTRF, homogeneous time-resolved fluorescence; MST, microscale thermophoresis.

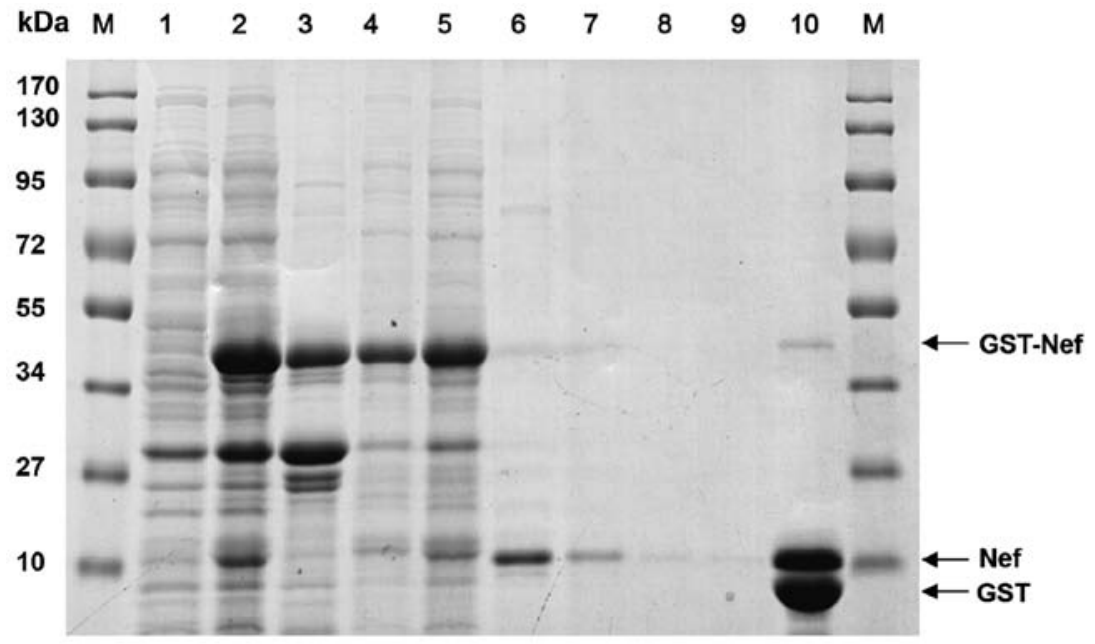

Figure 6. Affinity purification of Nef. Coomassie blue staining of the purification of Nef. Lane 1, E. coli BL21 transformed with pGEX-Nef without IPTG induction; lane 2, E. coli BL21 transformed with pGEX-Nef with IPTG induction; lane 3, the precipitation of ultrasound; lane 4, the supernatant of ultrasound after IPTG induction; lane 5, the supernatant of ultrasound flowed through the Glutathione Sepharose 4B; lane 6, the filtrate of GST-Nef fusion protein beads digested by thrombin; lane 7, the digested beads eluted with $3 \mathrm{ml}$ PBS first time; lane 8, the digested beads eluted with $3 \mathrm{ml}$ PBS second time; lane 9 , the digested beads eluted with $3 \mathrm{ml}$ PBS third time; lane 10, the beads after digested by thrombin and eluted by PBS.

result showed that the maxima of $\Delta \mathrm{F} \%$ appeared at $2.5 \mathrm{nM}$ anti-GST-Eu antibody, and the strongest interaction occurred at the anti-6xHis-XL665 antibody concentration $>40 \mathrm{nM}$ (Fig. 4C and D). To guarantee the effective detection of HTRF signal, the antibodies against GST-Eu and 6xHis-XL665 need to be excessive. In consideration of these factors, we chose respectively 2.5 and $100 \mathrm{nM}$ as the proper concentrations of anti-GST-Eu and anti-6xHis-XL665 antibodies for the following high-throughput screening.

High-throughput screening of the small molecules as inhibitors of Nef-sdAb19 interaction. After determination of the proper concentrations of four reagents for HTRF detection, $50 \mathrm{nM}$ GST-Nef, $20 \mathrm{nM}$ His-sdAb19, $2.5 \mathrm{nM}$ anti-GST-Eu and $100 \mathrm{nM}$ anti-6xHis-XL665 were used for screening the small molecular inhibitors against Nef-sdAb19 interaction. As described in Materials and methods, the high-throughput screening by HTRF tests were performed in 384-well plates in a total $50 \mu 1$ reaction volume containing the above reagents with their proper concentration and each small molecular compound $(100 \mu \mathrm{M})$ or control DMSO. After incubation, the 384-well plates were analyzed by Perkin-Elmer VICTOR $^{\mathrm{TM}}$ X5. The percentage of interaction inhibition was calculated with the formula: Inhibition $(\%)=\left(\left(\mathrm{F}_{\mathrm{Nef}}-\mathrm{F}_{\mathrm{GST}}\right)-\right.$ 

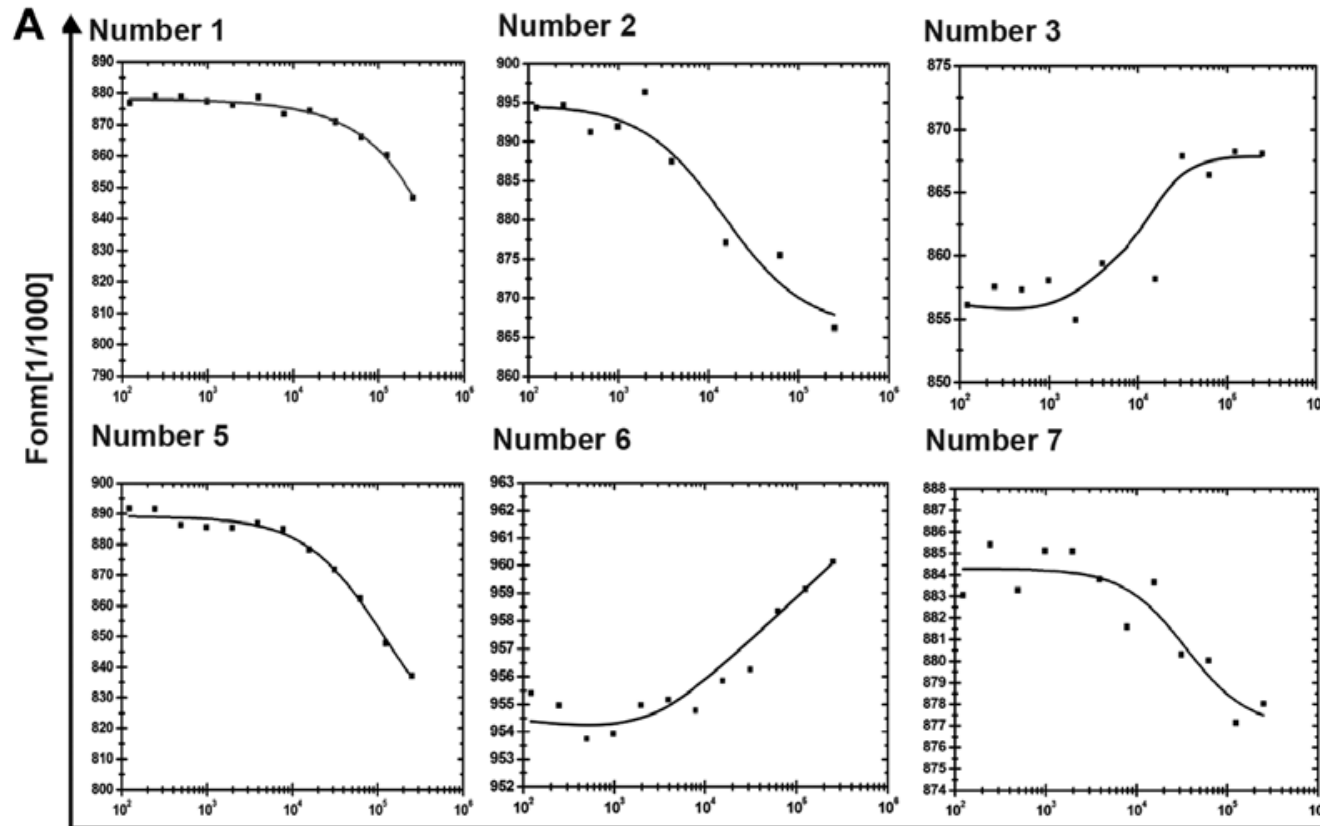

Number 4

Number 6

Number 7
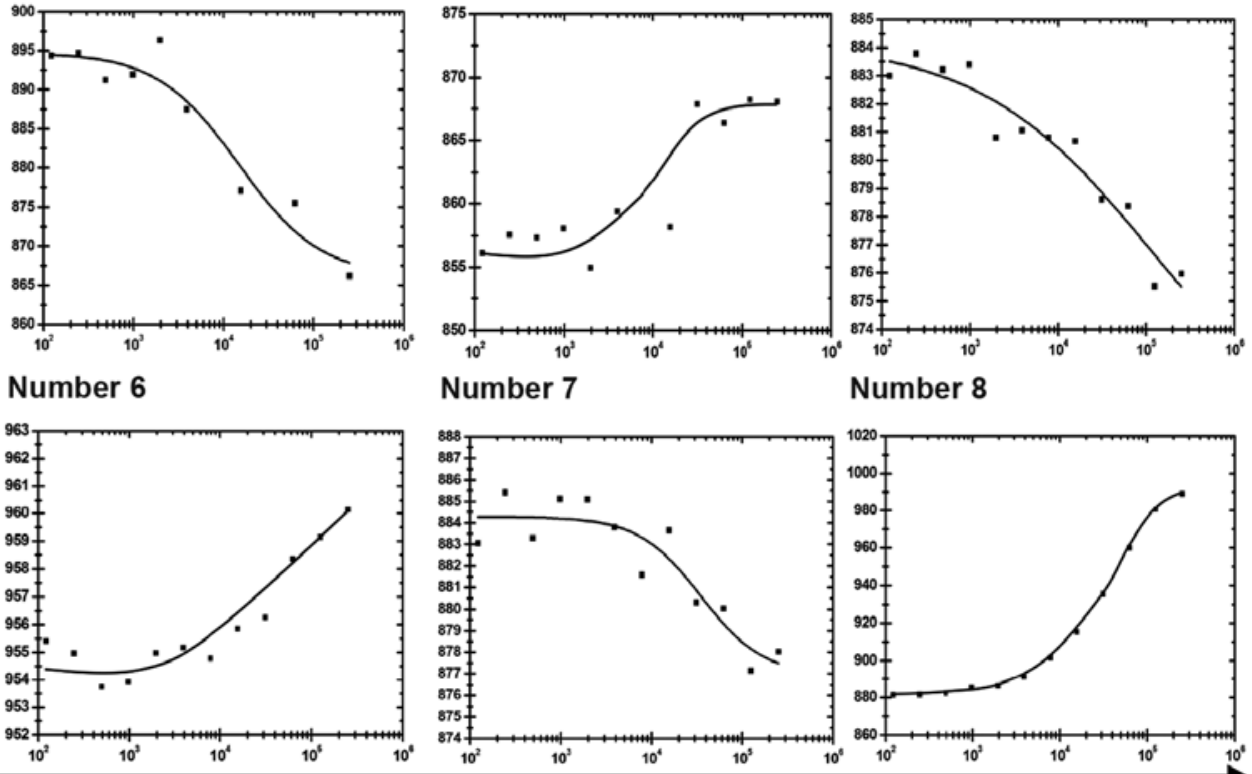

Number 8

Concentration

B

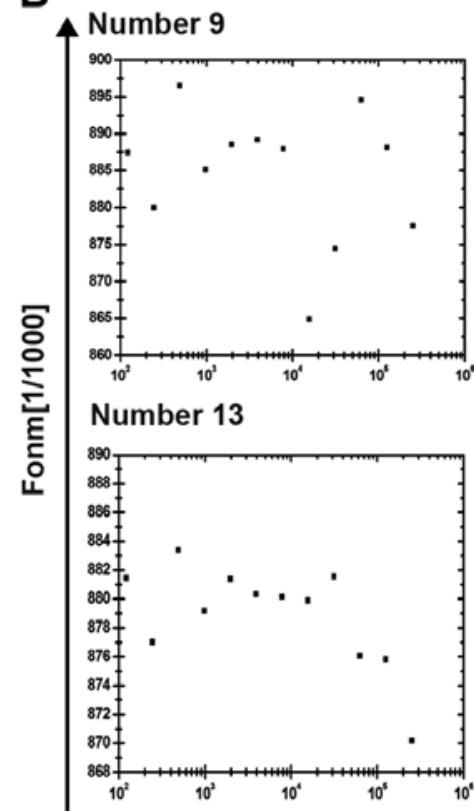

Number 10

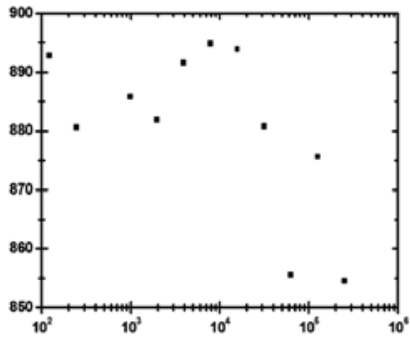

Number 14

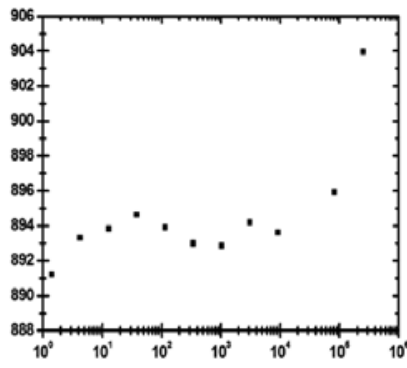

Number 11

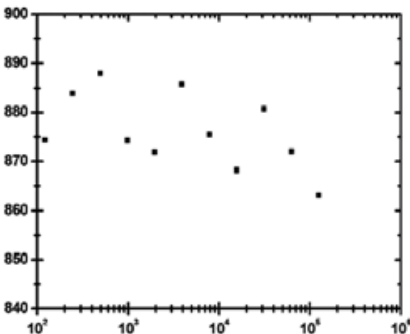

Number 15

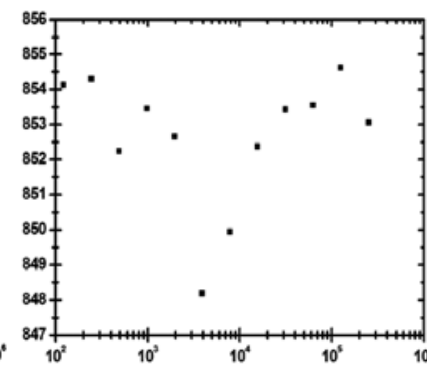

Number 12

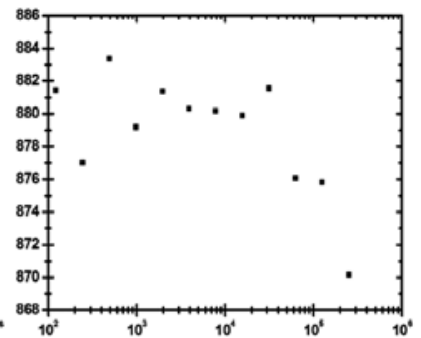

Number 16

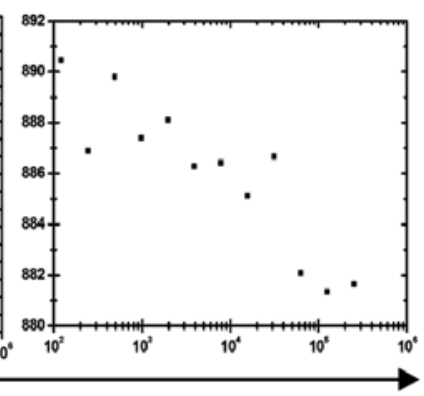

Concentration

Figure 7. Excluding the false-positive of HTRF using MST. The red fluorescent dye-labeled Nef was kept constant at $200 \mathrm{nM}$, and the sixteen candidate compounds were titrated from 100 to $25,000 \mathrm{nM}$, then plotted for analysis of thermophoresis and T-Jump. The KD is in a good agreement with literature values. (A) The positive MST; (B) The negative MST.

$\left.\left(\mathrm{F}_{\mathrm{com}}-\mathrm{F}_{\mathrm{GST}}\right)\right) /\left(\mathrm{F}_{\mathrm{Nef}}-\mathrm{F}_{\mathrm{GST}}\right) * 100$. The results in Fig. 5 showed that inhibition of most compounds was $<50 \%$, the generally acknowledged standard of effective inhibition in relation to high-throughput screening experiments. Notably, there were sixteen candidate compounds showing inhibition (\%) $>50 \%$ (Fig. 5). Their names and classes are shown in Table I).

Test of Nef binding by MST for HTRF-positive small molecules. In order to select among the candidate molecules that bind to Nef, we used microscale thermophoresis to test the interaction of each of the sixteen candidate molecules obtained and listed bove. Fig. 6 shows the SDS-PAGE of the recombinant Nef that we produced and purified for the MST assays. The red fluorescent dye-labeled Nef was kept constant at $200 \mathrm{nM}$, and the sixteen candidate compounds were titrated from 100 to $25,000 \mathrm{nM}$. The binding of Nef with the candidate compounds were monitored by the change in the thermophoretic property of the fluorescently labeled protein upon complex formation. The KD was caculated as previously reported (19). As shown by the MST T-Jump response in Fig. 7, there were eight candidate compounds that bind to Nef. 


\section{Discussion}

The protein Nef is critical for the pathogenesis of HIV, and the inhibition of its biological activity represents a potential way to eradicate HIV. The anti-Nef antibody sdAb19 derived from camelids significantly blocked the growth of HIV (23). Considering sdAb19 represent an efficient tool to elucidate the molecular functions of Nef, it is a meaningful strategy to search for new small molecular inhibitors against Nef-sdAb19 interaction for further inhibiting the function of Nef. In this study, we utilized HTRF technology to high-throughput screen the small molecular inhibitors against the interaction between Nef and sdAb19 in vitro. We obtained sixteen candidate compounds significantly inhibiting the interaction between Nef and sdAb19 through screening. Since the HTRF method that we used in our lab was inappropriate to monitor the interaction between Nef and small molecules, we used MST to select the Nef binding molecules, The results showed that only eight candidate compounds were positive for the binding with Nef in an extracellular context and represented therefore the candidate compounds for mimicking the antiNef activity of sdAb19 in vivo. The eight double-positive candidate molecules belong to 4 categories of compounds, including ion channel, phosphorylation, immunomodulators and antibiotics (Table I).

Ion channels are membrane proteins that are found in a number of viruses (21). In this study, felodipine and $\mathrm{R}(+)-I A A-94$ displaying potency in inhibition of ion channel activity (22) were selected for their ability of inhibiting Nef-sdAb19 interaction and binding to Nef. Protein phosphorylation is a reversible post-translational modification essential for the regulation of many cellular processes. Phosphorylation can modulate protein properties including enzymatic activity, stability, subcellular localization and interaction with binding partners. The importance of phosphorylation of the replication proteins of negative-strand RNA viruses has previously been documented (23). 3-[(2,4,6-trimethoxyphenyl)methylidenyl]indolin-2-one (IC261), a novel casein kinase-1 delta/epsilon ( $\delta / \varepsilon)$ specific inhibitor, triggers the mitotic checkpoint and induces p53-dependent postmitotic effects. The effects of IC261 on CK1 $1 \delta / \varepsilon$ might be the interference of specific phosphorylation of spindle component proteins (24). Notably, several recent studies showed that the CK1 $\delta / \varepsilon$ is involved in the regulation

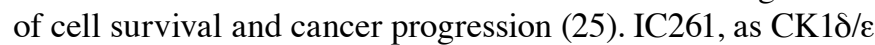
inhibitor, may inhibit cancer development through inhibition of microtubule polymerization (26). Immunomodulators and antibiotics are bringing great prospects for clinical trials, since there have been significant advances in immunotherapeutic field in HIV over the past decade (27). Retinoids, derivatives of vitamin A, have multiple cellular functions including induction of differentiation, regulation of apoptosis and inhibition of proliferation or inflammation (28). Retinoic acid p-hydroxyanilide is a vitamin A acid analog with antiproliferative activity in cultured human breast cancer cells $(29,30)$. Some studies found that all-trans retinoic acid (at RA) might inhibit HIV-1induced podocyte proliferation and dedifferentiation with the cAMP/PKA pathway through Nef-induced MAPK1,2 activation by retinoic acid receptor $\alpha(31,32)$.

The sdAb19 that derives from camelids, binds with high affinity $\left(\mathrm{Kd}=2 \times 10^{-9} \mathrm{M}\right)$ and shows specificity to the conserved epitope of Nef. The interaction between Nef and sdAb19 was confirmed by GST pull-down in our study. Small organic molecules could be revealed to be advantageous over antibody- or protein-based drugs due to their higher stability, bioavailability, deliverability and lower cost. We developed an HTRF-based screening assay for the identification of small molecule inhibitors against Nef-sdAb19 interaction that can be used also for larger scale screening. We performed HTRF assay with very low protein concentrations (Nef $50 \mathrm{nM}$; sdAb19 $25 \mathrm{nM}$ ) to increase the chances of finding small-molecule inhibitors. This is a novel trial for screening the antiviral small molecule drugs using the HTS assays. Sixteen candidate compounds were screened by HTRF assay, and eight compounds showed positive against Nef-sdAb19 interaction using MST.

In conclusion, the research presented in this study represents a proof of concept of a novel method for the screening of small molecule compounds, and permited the selection of 8 candidate molecules susceptible to inhibit the biological functions of HIV Nef protein and the physiopathogenesis of AIDS.

\section{Acknowledgements}

We thank Dr Liang Zhou (Quantum Design China \& NanoTemper Technologies $\mathrm{GmbH}$ ) for performing the Microscale Ther-mophoresis (MST) assay. This study was supported by the National Program on Key Basic Research Project (973 Program) (grant no. 2011CB910700), High-Level Talents Project of the Universities of Guangdong (grant no. [2011]431); National Natural Science Foundation of China (grant no. 31000628), Fundamental Research Funds for the Central Universities (grant nos. 21611430, 21610101 and 21609317), Natural Science Foundation of Guangdong Province (grant no. 9151065004000005$)$.

\section{References}

1. Greene WC: The brightening future of HIV therapeutics. Nat Immunol 5: 867-871, 2004.

2. Foster JL and Garcia JV: HIV-1 Nef: At the crossroads. Retrovirology 5: 84, 2008.

3. Kestler HW III, Ringler DJ, Mori K, Panicali DL, Sehgal PK, Daniel MD and Desrosiers RC: Importance of the nef gene for maintenance of high virus loads and for development of AIDS. Cell 65: 651-662,1991.

4. Fackler OT and Baur AS: Live and let die: Nef functions beyond HIV replication. Immunity 16: 493-497, 2002.

5. Shugars DC, Smith MS, Glueck DH, Nantermet PV, SeillierMoiseiwitsch F and Swanstrom R: Analysis of human immunodeficiency virus type 1 nef gene sequences present in vivo. J Virol 67: 4639-4650, 1993.

6. Kwak YT, Raney A, Kuo LS, Denial SJ, Temple BR, Garcia JV and Foster JL: Self-association of the Lentivirus protein, Nef. Retrovirology 7: 77, 2010.

7. Cruz NV, Amorim R, Oliveira FE, Speranza FA and Costa LJ: Mutations in the nef and vif genes associated with progression to AIDS in elite controller and slow-progressor patients. J Med Virol 85: 563-574, 2013.

8. Foster JL, Denial SJ, Temple BR and Garcia JV: Mechanisms of HIV-1 Nef function and intracellular signaling. J Neuroimmune Pharmacol 6: 230-246, 2011.

9. Bouchet J, Basmaciogullari SE, Chrobak P, Stolp B, Bouchard N, Fackler OT, Chames P, Jolicoeur P, Benichou S and Baty D: Inhibition of the Nef regulatory protein of HIV-1 by a singledomain antibody. Blood 117: 3559-3568, 2011.

10. Muyldermans S: Single domain camel antibodies: Current status. J Biotechnol 74: 277-302, 2001 
11. Roovers RC, Laeremans T, Huang L, De Taeye S, Verkleij AJ, Revets H, de Haard HJ and van Bergen en Henegouwen PM: Efficient inhibition of EGFR signaling and of tumour growth by antagonistic anti-EFGR Nanobodies. Cancer Immunol Immunother 56: 303-317, 2007.

12. Gueorguieva D, Li S, Walsh N, Mukerji A, Tanha J and Pandey S: Identification of single-domain, Bax-specific intrabodies that confer resistance to mammalian cells against oxidative-stressinduced apoptosis. FASEB J 20: 2636-2638, 2006.

13. Hultberg A, Temperton NJ, Rosseels V, Koenders M, Gonzalez-Pajuelo M, Schepens B, Ibañez LI, Vanlandschoot P, Schillemans J, Saunders M, et al: Llama-derived single domain antibodies to build multivalent, superpotent and broadened neutralizing anti-viral molecules. PLoS One 6: e17665, 2011

14. Ohmi N, Wingfield JM, Yazawa H and Inagaki O: Development of a homogeneous time-resolved fluorescence assay for high throughput screening to identify Lck inhibitors: Comparison with scintillation proximity assay and streptavidin-coated plate assay. J Biomol Screen 5: 463-470, 2000.

15. Degorce F: HTRF $^{\circledR}$ : Pioneering technology for high-throughput screening. Expert Opin Drug Discov 1: 753-764, 2006.

16. Jia Y, Quinn CM, Gagnon AI and Talanian R: Homogeneous time-resolved fluorescence and its applications for kinase assays in drug discovery. Anal Biochem 356: 273-281, 2006.

17. Chen X, Longgood JC, Michnoff C, Wei S, Frantz DE and Bezprozvanny L: High-throughput screen for small molecule inhibitors of Mint1-PDZ domains. Assay Drug Dev Technol 5: 769-783, 2007.

18. Mohamed TM, Zakeri SA, Baudoin F, Wolf M, Oceandy D, Cartwright EJ, Gul S and Neyses L: Optimisation and validation of a high throughput screening compatible assay to identify inhibitors of the plasma membrane calcium ATPase pump - a novel therapeutic target for contraception and malaria. J Pharm Pharm Sci 16: 217-230, 2013.

19. Lemay J, Maidou-Peindara P, Bader T, Ennifar E, Rain JC, Benarous R and Liu LX: HuR interacts with human immunodeficiency virus type 1 reverse transcriptase, and modulates reverse transcription in infected cells. Retrovirology 5: 47, 2008.

20. Seidel SA, Dijkman PM, Lea WA, van den Bogaart G, JerabekWillemsen M, Lazic A, Joseph JS, Srinivasan P, Baaske P, Simeonov A, et al: Microscale thermophoresis quantifies biomolecular interactions under previously challenging conditions. Methods 59: 301-315, 2013
21. Fischer WB, Wang YT, Schindler C and Chen CP: Mechanism of function of viral channel proteins and implications for drug development. Int Rev Cell Mol Biol 294: 259-321, 2012.

22. Izzedine H, Launay-Vacher V, Deray G and Hulot JS: Nelfinavir and felodipine: A cytochrome P450 3A4-mediated drug interaction. Clin Pharmacol Ther 75: 362-363, 2004.

23. Idriss HT: Questions regarding the role of protein phosphorylation in HIV replication and anti-acquired immune deficiency syndrome therapy. Saudi Med J 26: 7-10, 2005.

24. Jakubiec A and Jupin I: Regulation of positive-strand RNA virus replication: The emerging role of phosphorylation. Virus Res 129: 73-79, 2007.

25. Behrend L, Milne DM, Stöter M, Deppert W, Campbell LE, Meek DW and Knippschild U: IC261, a specific inhibitor of the protein kinases casein kinase 1-delta and -epsilon, triggers the mitotic checkpoint and induces p53-dependent postmitotic effects. Oncogene 19: 5303-5313, 2000.

26. Yang WS and Stockwell BR: Inhibition of casein kinase 1-epsilon induces cancer-cell-selective, PERIOD2-dependent growth arrest. Genome Biol 9: R92, 2008.

27. Zhou YX, Karle S, Taguchi H, Planque S, Nishiyama Y and Paul S: Prospects for immunotherapeutic proteolytic antibodies. J Immunol Methods 269: 257-268, 2002.

28. Evans TR and Kaye SB: Retinoids: Present role and future potential. Br J Cancer 80: 1-8, 1999.

29. Kaleagasioglu F, Doepner G, Biesalski HK and Berger MR: Antiproliferative activity of retinoic acid and some novel retinoid derivatives in breast and colorectal cancer cell lines of human origin. Arzneimittelforschung 43: 487-490, 1993.

30. Hua S, Kittler R and White KP: Genomic antagonism between retinoic acid and estrogen signaling in breast cancer. Cell 137: 1259-1271, 2009.

31. He JC, Lu TC, Fleet M, Sunamoto M, Husain M, Fang W, Neves S, Chen Y, Shankland S, Iyengar R, et al: Retinoic acid inhibits HIV-1-induced podocyte proliferation through the cAMP pathway. J Am Soc Nephrol 18: 93-102, 2007.

32. Lu TC, Wang Z, Feng X, Chuang P, Fang W, Chen Y, Neves $S$, Maayan A, Xiong H, Liu Y, et al: Retinoic acid utilizes CREB and USF1 in a transcriptional feed-forward loop in order to stimulate MKP1 expression in human immunodeficiency virusinfected podocytes. Mol Cell Biol 28: 5785-5794, 2008. 